\title{
Miranda
}

Revue pluridisciplinaire du monde anglophone /

Multidisciplinary peer-reviewed journal on the English-

speaking world

$6 \mid 2012$

Marking the Land in North America

\section{Llewellyn Brown (éd.), Sam Beckett 1 : L'ascèse du sujet}

\section{Philippe Birgy}

\section{QpenEdition}

\section{Journals}

Édition électronique

URL : http://journals.openedition.org/miranda/3016

DOI : 10.4000/miranda.3016

ISSN : 2108-6559

Éditeur

Université Toulouse - Jean Jaurès

\section{Référence électronique}

Philippe Birgy, «Llewellyn Brown (éd.), Sam Beckett 7 : L'ascèse du sujet », Miranda [En ligne], 6 | 2012, mis en ligne le 28 juin 2012, consulté le 16 février 2021. URL : http://journals.openedition.org/miranda/ 3016 ; DOI : https://doi.org/10.4000/miranda.3016

Ce document a été généré automatiquement le 16 février 2021.

\section{$(1) \Theta$}

Miranda is licensed under a Creative Commons Attribution-NonCommercial-NoDerivatives 4.0 International License. 


\title{
Llewellyn Brown (éd.), Sam Beckett 1 : L'ascèse du sujet
}

\author{
Philippe Birgy
}

\section{RÉFÉRENCE}

Llewellyn Brown (éd.), Sam Beckett 1 : L'ascèse du sujet, La Revue des Lettres Modernes (Caen : Lettres Modernes Minard, 2010), 196 p, ISBN 978-2-256-91162-0

1 Ce recueil de textes sélectionnés et présentés par Llewellyn Brown, publié dans la collection La Revue des Lettres Modernes, frappe au premier abord par sa belle facture. Il ne s'agit pas seulement d'une nouvelle parution - laquelle mériterait déjà en soi toute l'attention des beckettiens - mais du premier numéro d'une série consacrée à cet auteur. Comme L. Brown le remarque dans sa préface, cette série vient combler une lacune puisque la production critique sur les œuvres de Beckett est largement le fait de chercheurs anglophones et/ou anglicistes. En conséquence, cette dernière se concentre en priorité sur les textes anglais de l'auteur. Mais il ne s'agit pas pour autant ici d'exclure la réflexion anglo-saxonne. Pour preuve la présence d'un article d'Anthony Uhlman, garant de cette ouverture à la critique étrangère (et plus généralement du sérieux de l'entreprise).

2 Ce premier volume s'intitule donc L'ascèse du sujet, et conformément au programme annoncé par le titre, il rassemble une série d'articles se rapportant à la question de l'ascétisme, entendu comme « diminution de la présence du sujet au monde » (6). Il est divisé en trois parties respectivement nommées "L'ascétisme et le corps ", «Le désir pur d'en finir » et «Un sujet ineffaçable », soit trois manières d'approche qui balisent et coordonnent efficacement les différentes contributions.

3 L'ascèse du sujet s'ouvre sur un exercice de comparatisme visant à estimer la pertinence d'un certain héritage grec. N. Larajinha reconnaît ainsi dans le phénomène de maximisation de l'activité de l'esprit, quand le corps cesse d'être opérant, une position platonicienne formulée dans le Phédon. 
4 Katerina Kanelli, toujours dans cette même perspective comparatiste, se fixe pour objectif d'estimer, dans une brève séries de notes de lectures, le traitement du mouvement dans Mal vu mal dit de Beckett et Ascèse de Salvatores Deu, ceci afin de mieux faire apparaître la singularité d'un usage beckettien.

Clara Montini, dans un article à thèse précisément documenté, observe comment la traduction du français vers l'anglais participe de la mise à distance de la corporalité chez Beckett. S'appuyant principalement sur les théories lacaniennes, elle entend démontrer que, chez l'auteur, « le corps du texte en vient à remplacer le sujet » (39).

Anthony Uhlmann, que les spécialistes connaissent pour ses travaux sur les relations entre Beckett et la philosophie, assoit avec autorité l'hypothèse d'une filiation présocratique dans les récits de l'auteur. Raisonnant également à partir des arguments posés par une lignée de philosophes néoplatoniciens ainsi que par les rationalistes qui s'en inspirent, A. Uhlmann se confronte à la question de l'être, de ses limites et de son unité, telle qu'elle se donne à penser dans Mal vu mal dit et Molloy.

7 La contribution de Dirk Van Hulle se présente comme un essai de critique génétique. De nombreux documents d'archive y sont mis à contribution pour accéder à une problématique d'auteur. Ainsi, D. Van Hulle déduit des corrections et des omissions de l'auteur dans les épreuves et les manuscrits que la considération du suicide, refoulée dans ses textes, "s'avère plus importante [pour Beckett] comme motif créatif que comme thème explicite».

8 Franz Kaltenbeck se livre à une lecture psychanalytique de A Piece of Monologue et Solo, afin d'y souligner la prédominance d'une réflexion sur les enjeux de la mortalité et d'avérer son impact déterminant sur l'écriture beckettienne. C'est selon Beckett « une des fonctions principales de la poésie » que de "parler des mourants et des morts ». F. Kaltenbeck insiste sur le caractère vital de l'obstination des personnages beckettiens à ne pas se séparer des disparus, à tenir coûte que coûte aux êtres défunts. Partant de la formule "Birth was the death of me ", il souligne le processus d'extension du problème de la mort qui en vient à recouvrir l'existence toute entière. En effet, ce dernier se pose dès la naissance, celle-ci étant équivalente à la " perte » (87).

9 Nadia Louar développe une ambitieuse réflexion générique à propos de la notion de style et de son dépassement dans l'œuvre de Beckett. Reprenant l'objectif joycéen d'une langue sans style, Beckett semble avoir visé ce même dépouillement en "esquivant l'être et le dire ", en essayant "d'enrayer tout ancrage dans la langue » de façon à émanciper l'auteur de «toutes les assignations identitaires fixes ». La proposition de N. Louar s'accompagne d'une définition de travail du style. Elle nous invite ainsi à le considérer comme automatisme plutôt que comme choix de l'écrivain. D'où la difficulté à s'en défaire "sans s'annuler en tant qu'auteur » (111). N. Louar détaille alors par l'exemple une stylistique de l'absence et documente ce qu'elle nomme la "génération de nouveaux codes sémantiques".

10 Isabelle Ost voit dans l'œuvre de Beckett l'écriture d'un désir évasif et sans fin, produit de la machine-texte, qui ne se manifeste que par l'évidemment du sujet. Elle nous invite ainsi à porter notre attention sur l'aspect machinal des scènes et des narrations beckettiennes. C'est donc une lecture fondamentalement deleuzienne qu'elle expose.

11 Enfin, l'article de Llewellyn Brown se donne pour objectif critique de «creuser la nature du désir ", tel qu'il est figuré chez Beckett. L. Brown distingue une première période dans l'œuvre, laquelle reste encore tributaire d'une conception du désir- 
manque, présupposant un Autre capable de combler les attentes du sujet. Or, dans la deuxième partie de sa carrière, Beckett en vient à confronter ses personnages à «la question existentielle qui se pose à l'endroit où l'Autre fait radicalement défaut » (159). Il suggère ainsi que les formules beckettiennes de l'absence radicale - l'impossible, l'innommable - sont davantage que de simples privatifs: elles sont «le roc de l'existence».

L'avantage certain d'une série sur une publication isolée est qu'elle permet de mieux cibler le périmètre d'investigation, et consécutivement de favoriser des analyses plus fines. Et l'on remarque en effet que le partage entre théorie et observation textuelle ne se fait jamais à l'exclusion de l'une ou de l'autre, mais que les deux aspects critiques s'équilibrent au fil les articles. L'ascèse du sujet est une réussite à cet égard. Certes, ou pourrait éventuellement craindre que la prédominance des approches deleuzienne et lacanienne puisse refermer indûment le champ de la réflexion. Cependant, cette prédominance est toute relative. Outre que les deux penseurs cités sont bien loin de pouvoir servir de base à un quelconque consensus critique, le recours à d'autres grilles contribue à entretenir un débat contradictoire. A l'inverse, on ne pourra pas prétendre que les articles élaborent un plan de réflexion totalement homogène et cohérent: il convient de s'en féliciter, car une telle unité présenterait un intérêt moindre, en comparaison de la variété des perspectives offertes ici.

Ajoutons encore que l'ouvrage est complété par une section de résumés des articles, ainsi que par quatorze pages de comptes rendus de lectures, auxquels s'ajoute la table des matières du deuxième numéro de la même série, d'ores et déjà disponible, et il sera certainement apparu, au terme de ce compte-rendu, que Sam Beckett 1: L'ascèse du sujet devrait retenir l'intérêt des beckettiens francisants et/ou anglicistes.

\section{INDEX}

Mots-clés : absence, ascétisme, corps, désir, esprit, Fin de partie, mort, philosophie, psychanalyse, théâtre

Keywords : absence, asceticism, body, desire, death, drama, Endgame, Molloy, Phaedon, philosophy, psychoanalysis, solo, spirit

\section{AUTEURS}

\section{PHILIPPE BIRGY}

Professeur

Université Toulouse 2 - Le Mirail

birgy@univ-tlse2.fr 